\title{
Implantable cardioverter defibrillators: therapy against Canada's leading killer
}

ß See related article page 1053

4 very year, sudden cardiac 4 death claims the lives of 45000 Canadians. This translates into 120 deaths a day, or 1 death every 14 minutes - a higher toll than is taken by lung cancer, stroke, AIDs and breast cancer combined. ${ }^{1}$

Implanted cardioverter defibrillators (ICDs) were developed in the early 1980s as prophylaxis against sudden cardiac death. Early rudimentary devices weighed up to $300 \mathrm{~g}$, had a battery life of 3 years and were implanted in the abdomen with epicardial leads sewn directly onto the heart. Current devices weigh less than $80 \mathrm{~g}$ and have a battery life of 7 years. They consist of 2 components: a pulse generator and endocardial leads. The pulse generator consists of a battery, a high-voltage capacitor, bradycardia/tachycardia detection circuitry and pacing circuitry. Pulse generators are implanted into the subcutaneous tissue below the clavicle and are attached to transvenous leads that lead into the right-sided chambers. Transvenous leads are the means by which the pulse generator senses local myocardial activations, delivers pacing stimuli and, ultimately, delivers defibrillation shocks. Pulse generators are designed for single (right ventricle only), dual (right atrium and ventricle) or triple (right atrium and ventricle and left ventricle) chamber leads, depending on the clinical indication.

ICDs are capable of detecting and stopping tachyarrhythmias. Detection of the arrhythmia is guided primarily by heart rate: the interval between successive stimulations of the implanted lead is averaged over a few beats. If the heart rate exceeds a specified threshold, the device initiates a heart rate- specific therapy. Most devices are programmed with 2 heartrate zones: the ventricular fibrillation (VF) zone and the ventricular tachycardia (VT) zone. In the VT zone (usually programmed for heart rates between 150 and 200 beats per minute), the device will attempt to pace the ventricle out of the malignant rhythm. If this antitachycardia pacing fails to terminate the arrhythmia, the device will deliver a shock to return the patient's rhythm back to sinus. In contrast, arrhythmias that fall into the $\mathrm{VF}$ zone (usually programmed for heart rates above 200 beats per minute) are immediately shocked back into sinus rhythm.

\section{Who should get an ICD?}

Although sudden cardiac death can be the first manifestation of cardiac disease in approximately

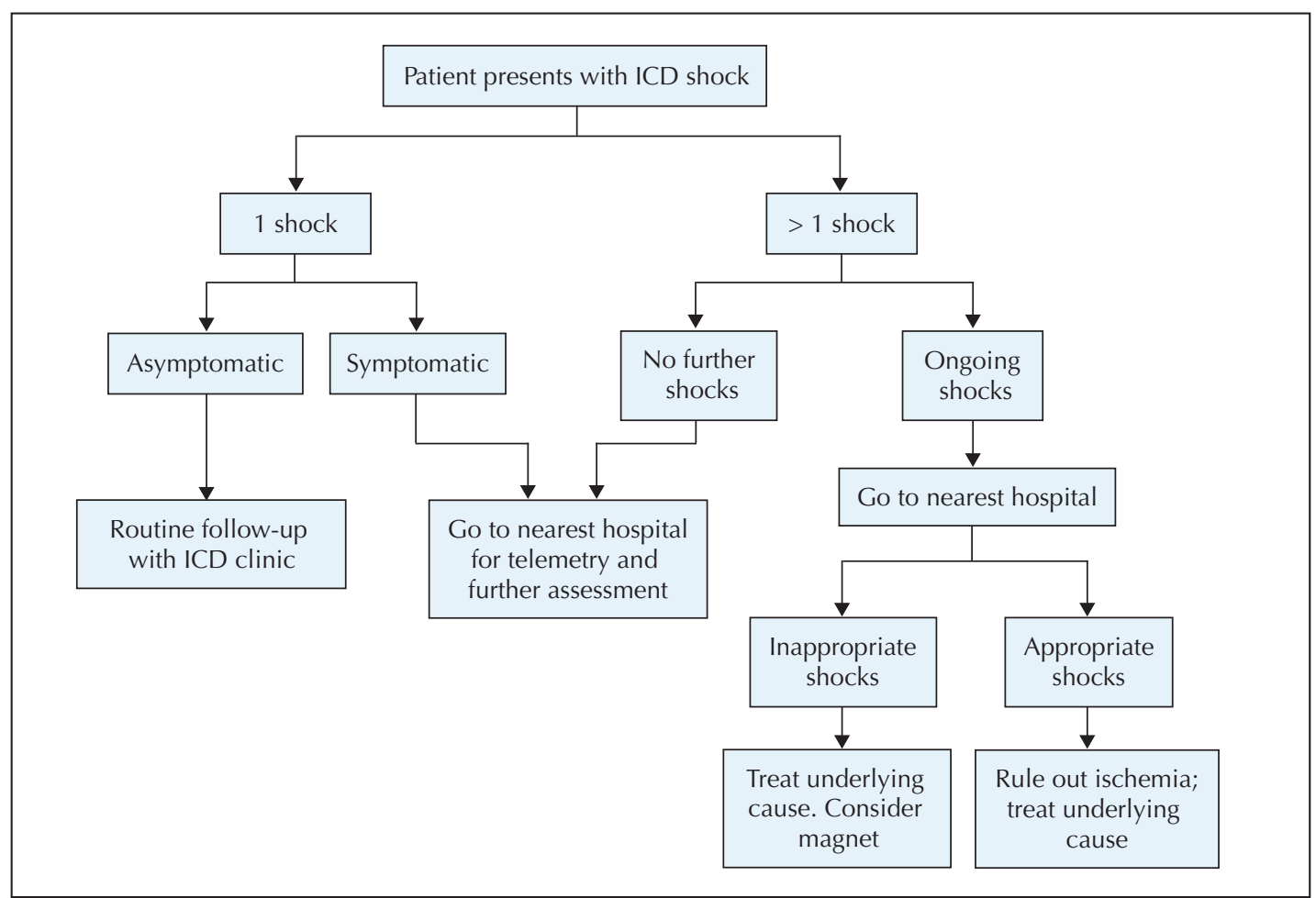

Fig. 1: Summary of treatment algorithm for patients whose implantable cardioverter defibrillator (ICD) is delivering shocks. 
$20 \%$ to $25 \%$ of cases, it usually occurs in patients with clinically recognized heart disease. The rate of sudden cardiac death among patients with a history of myocardial infarction is 4 to 6 times that in the general population. Among patients with heart failure or left ventricular dysfunction, the sudden cardiac death rate is 6 to 9 times higher than in the general population.

ICDs were originally designed for survivors of clinical ventricular arrhythmia/tachycardia or cardiac arrest (secondary prevention). Several clinical trials have shown that ICDs significantly improve overall survival (number needed to treat [NNT] 9 to 24). ${ }^{2}$ This is reflected in the current guidelines for the use of ICDs.

However, most patients (95\%) who present with a malignant ventricular arrhythmia do not survive. Therefore, rather than reserving ICDs for those who have had a cardiac arrest, it would seem logical to identify those patients who are at high risk for sudden cardiac death and use an ICD for primary prevention. Several clinical trials have shown that patients with ischemic cardiomyopathy and an ejection fraction of less than $30 \%$ derive an all-cause mortality benefit from ICD therapy (NNT 9.1). ${ }^{3}$ More recently the SCD-HeFT trial showed that these benefits extended to patients with heart failure from any cause with an LV ejection fraction $\leq 35 \%$ (NNT 1). Other patients at risk for sudden cardiac death have been identified using current stratification techniques for hereditary conditions such as arrhythmogenic right ventricular cardiomyopathy, Brugada's syndrome, hypertrophic cardiomyopathy and short/long QT syndrome.

\section{When the ICD fires}

Some patients may present to their primary care physician af- ter receiving an ICD shock. If the patient is feeling well with no recurrent shock, a routine follow-up with the ICD clinic will suffice. However, if the patient experiences several shocks in succession, admission to the nearest hospital may be required for stabilization and investigation of potential reversible causes (i.e., ischemia, electrolyte or metabolic abnormalities) (see Fig. 1).

Because of the effectiveness of ICD therapy, more patients using these devices are surviving longer and may present with recurrent appropriate ICD shocks - that is, "electrical storm." One of the most common causes of electrical storm is ischemia; in such cases urgent revascularization should be considered. Patients without obvious anginal ischemia should be stabilized and treated with appropriate antiarrhythmic medication (i.e., magnesium, $\beta$ blockers and amiodarone). Reversible causes should be identified and treated (i.e., hypokalemia, hypocalcemia, hypomagnesemia, drugs). All patients with recurrent ventricular arrhythmias should be sedated, as shocks delivered by the ICD are painful. Once the patient is medically stabilized, transfer to a tertiary care institution should be considered. These arrhythmias may be amenable to programming optimization (i.e., aggressive anti-tachycardic pacing protocols) or invasive procedures (i.e., electrophysiology study and ablation of ventricular tachycardia or fibrillation).

Occasionally, patients with ICDs present with recurrent inappropriate shocks caused by supraventricular arrhythmia, oversensing of the $\mathrm{T}$ wave or electrical noise. This condition is often suspected only after a shock event is captured on telemetry, showing either a new-onset tachyarrhythmia followed by a shock (SVT-shock) or a shock occurring in sinus rhythm (oversensed $\mathrm{T}$ wave or electrical noise). Manufacturers of ICDs have attempted to improve the ability of their devices to differentiate ventricular arrhythmias from other tachyarrhythmias by permitting the devices to analyze factors such as atrial-ventricular relationships, electrocardiogram morphology, stability of the rate and onset of the arrhythmia before delivery of a shock. If inappropriate shocks are suspected, application of a strong magnet over the device will flip an internal switch that puts the defibrillator's shock functions on standby. It is important to note that the ICD will continue to function as a back-up pacemaker should the patient's sinus rate drop below the specified lower rate limit.

Sudden cardiac death represents a real and sizeable hazard to Canadians. As research trials continue to characterize risk factors for sudden cardiac death, and increasing numbers of patients are offered prophylactic ICD therapy, it is important for primary care physicians to become familiar with how these devices work, the clinical contexts in which they are used and what to do when patients report that their ICD is firing.

\section{Darryl R. Davis}

Anthony S.L. Tang

Division of Cardiology

University of Ottawa Heart Institute

Ottawa, Ont.

\section{References}

1. Gardner MJ, Leather R, Teo K. Prevention of sudden death from ventricular arrhythmia. Epidemiology. Can $\mathcal{7}$ Cardiol 2000;16(Suppl C):10C-12C.

2. Connolly SJ, Hallstrom AP, Cappato R, Schron EB, Kuck KH, Zipes DP, et al. Meta-analysis of the Implantable Cardioverter Defibrillator Secondary Prevention Trials. AVID, CASH and CIDS Studies. Antiarrhythmics Vs Implantable Defibrillator Study. Cardiac Arrest Study Hamburg. Canadian Implantable Defibrillator Study. Eur Heart 7 2000;21(24):2071-8.

3. Moss AJ, Zareba W, Hall WJ, Klein $\mathrm{H}$, Wilber DJ, Cannom DS, et al. Prophylactic implantation of a defibrillator in patients with myocardial infarction and reduced ejection fraction. N Engl f Med 2002;346(12):877-83. 